\title{
Going against the flow: maintenance of alongshore variation in allele frequency in a coastal ocean
}

\author{
James M. Pringle ${ }^{1, *}$, John P. Wares ${ }^{2}$ \\ ${ }^{1}$ Department of Earth Sciences, and the Institute of Earth, Oceans \& Space, University of New Hampshire, Durham, \\ New Hampshire 03824, USA \\ ${ }^{2}$ Department of Genetics, Life Science Building, University of Georgia, Athens, Georgia 30602, USA
}

\begin{abstract}
Understanding the processes that develop and maintain diversity in coastal communities is an important challenge, particularly given the conservation and management needs of these ecosystems. Such diversity patterns include not only species distributions and interactions, but also variation in genetic diversity. Alongshore variations in allele frequency along coastal oceans are controlled by interactions between physical and biological forces. Here we model these interactions in terms of Lagrangian descriptors of nearshore physical oceanography, the life history dynamics of an individual species and habitat quality. This model allows us to define population boundaries within the species range as a function of ocean circulation, spatial habitat variability and larval characteristics. From this, we can find quantitative criteria for the persistence of regions of alongshore genetic variation. Our results show quantitatively that the existence of phylogeographic patterns in species with high dispersal capacity along uniform coasts with typical mean currents is surprising, and requires either strong selection gradients, alongshore variation in ocean currents and/or habitat quality, or a mixture of both. Our model suggests that marine reserves and the harvesting of marine recources can dramatically modify spatial gradients in genetic diversity.
\end{abstract}

KEY WORDS: Advection · Coastal $\cdot$ Phylogeography $\cdot$ Cline $\cdot$ Retention

\section{INTRODUCTION}

A classic problem in the study of marine biodiversity is how patterns of genetic differentiation arise in an environment with few apparent barriers to dispersal, in species with high capacity for dispersal due to lar$\mathrm{val} / \mathrm{juvenile} \mathrm{life} \mathrm{history,} \mathrm{and} \mathrm{in} \mathrm{regions} \mathrm{where} \mathrm{coastal}$ currents should promote population mixing (Palumbi 1994). Most phylogeographic studies of coastal species emphasize the historical changes that could have initiated allopatry and the process of genetic differentiation. Little attention is generally given to the processes that then must maintain spatial genetic discontinuities in these populations, except in situations where physiological or other barriers are apparent, e.g. the Florida peninsula preventing gene flow between the Gulf and Atlantic lineages (Avise 1994). Here we quantitatively examine what processes allow spatial genetic disconti- nuities to persist in a coastal ocean for species with a planktonic dispersal stage. Some and possibly most of these patterns, including gradual clines and phylogeographic 'breaks' (see Avise 2000), may reflect the disparity between migration and gene flow caused by selection (Barton \& Hewitt 1989, Palumbi 1994, Sotka et al. 2003, Hare et al. 2005).

Many phylogeographic studies involve an inherent assumption that the gene flow process being described is selectively neutral, which is to say that the pattern is evidence of dispersal limitation alone, rather than incorporating factors that limit gene flow, such as selection or ecological interactions, as well as stochastic factors associated with historical vicariance and the genealogical data themselves (Haydon et al. 1994, Irwin 2002). Here we present a model that describes the interaction between selection, defined by the relative reproductive success of individuals, and physical 
oceanography. We find that it is unlikely that a cline can persist in species with high dispersive capacity in an advective environment for an ecologically significant time without selection favoring the downstream allele, where downstream is defined with respect to the mean direction of larval transport.

We find that there are 2 inter-related mechanisms by which a cline or other phylogeographic pattern (hereafter, any population genetic discontinuity among regions is referred to as a cline) can persist in the coastal ocean in the face of non-random dispersal of larvae. Firstly, a spatial gradient in selection can be sufficient to allow a cline to persist (Endler 1977, Hare et al. 2005). We extend prior results by quantifying the strength of the selection gradient needed to allow a cline to persist as a function of larval characteristics and local oceanographic conditions. Secondly, alongshore variability in habitat quality or larval transport can create a region that is recruit-limited, leading to increased local reproductive success and population retention. By explicitly including spatial variability in population dynamics in our model, we find that these regions can allow a cline to persist at lower selection levels than in the absence of alongshore variation.

Our model allows earlier results of Nagylaki (1978) and others who studied systems with biased directional dispersal to be applied to a coastal ocean without a priori demarcation of the spatial extent of populations.

Table 1. Definitions of parameters used in the model

\begin{tabular}{|c|c|}
\hline Parameter & Definition \\
\hline$f_{\text {migrate }}$ & $\begin{array}{l}\text { Fractional change in larval transport from upstream, } \\
\text { e.g. } f_{\text {migrate }}=0.2 \text { denotes that transport of larvae from upstream } \\
\text { of a point is } 1 / 5 \text { of what it is elsewhere }\end{array}$ \\
\hline$\gamma$ & $\begin{array}{l}\text { Fraction of habitat not occupied by adults. If larvae exhibit } \\
\text { avoidance behavior and do not settle on occupied habitat, } \gamma=1\end{array}$ \\
\hline$H_{\text {dens }}$ & Maximum population per unit length of habitat \\
\hline$L_{\mathrm{adv}}$ & Mean distance larvae recruits downstream of parents \\
\hline$L_{\text {diff }}$ & $\begin{array}{l}\text { Standard deviation of distance larvae recruits downstream } \\
\text { of parents }\end{array}$ \\
\hline$m$ & $\begin{array}{l}\text { Fraction of larvae in retention zone entering from upstream } \\
\text { of the zone }\end{array}$ \\
\hline$N_{\text {allele }}$ & $\begin{array}{l}\text { Number of additional copies of an allele in population for each } \\
\text { existing copy of allele in population a lifetime earlier }\end{array}$ \\
\hline$N_{\text {larvae }}$ & $\begin{array}{l}\text { Number of larvae adult produces per lifetime capable of re- } \\
\text { recruiting successfully and reaching reproductive age, if habitat } \\
\text { available, net of all mortality in larval pool and after settlement }\end{array}$ \\
\hline$s$ & $\begin{array}{l}\text { Selection against all alleles except allele of interest } \\
\text { (see Hartl \& Clark 1997) }\end{array}$ \\
\hline$\sigma$ & $\begin{array}{l}\text { Standard deviation of Lagrangian alongshore currents } \\
\text { experienced by planktonic larvae }\end{array}$ \\
\hline$\tau_{\mathrm{L}}$ & $\begin{array}{l}\text { Lagrangian timescale of alongshore currents experienced } \\
\text { by planktonic larvae }\end{array}$ \\
\hline$T_{\mathrm{m}}$ & Planktonic larval duration \\
\hline$U$ & Mean alongshore current speed experienced by planktonic larvae \\
\hline
\end{tabular}

Through analysis of the effect of population dynamics on the retention of alleles one can discover how to define populations (demes) in such a system, identify 'source' and 'sink' populations, and show how these populations are related to the oceanic circulation that drives dispersal and alongshore variation in habitat quality. Without such a quantitative description of how abiotic forces interact to maintain geographic variation in genetic markers, we are left with only a static view of how allelic diversity is regulated in natural communities. This model shows how observed spatial patterns of genetic differentiation arise from the interaction of ocean circulation, larval dispersal and spatial variation in habitat quality.

\section{METHODS}

Model of a benthic species with planktonic dispersal. The theoretical results derived in the present study are obtained for an idealized model of a coastal benthic organism with a planktonic larval stage. The retention of a population of this idealized species in the presence of mean and variable alongshore flows has been described in Byers \& Pringle (2006). In this section we describe this organism and a simple numerical model that describes its dispersal and population dynamics. Each adult produces $N_{\text {larvae }}$ offspring per lifetime that can recruit and grow to reproductively competent adulthood, if there is sufficient empty habitat for the larvae to settle in; it is important to note that

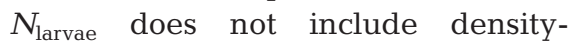
dependent mortality effects caused by habitat scarcity at settlement, but it incorporates mortality in the larval pool and after settlement but before reproductive competency. The organism is assumed to live in a coastal habitat narrow enough that its larvae can spread across the cross-shelf dimension of the habitat in a single generation, so that the habitat can be treated as one-dimensional. The habitat has a carrying capacity of $H_{\text {dens }}$ adults $\mathrm{m}^{-1}$. These and all other parameters are defined in Table 1.

Each larva is dispersed a variable distance downstream from its parent. The mean dispersal distance is $L_{\mathrm{adv}}$ and the standard deviation of the dispersal distance is $L_{\text {diff }}$ (see Fig. 1). For simple circulation and behavioral regimes, $L_{\mathrm{adv}}$ is the distance the larvae are advected by the mean currents and $L_{\text {diff }}$ is the distance that eddies and 


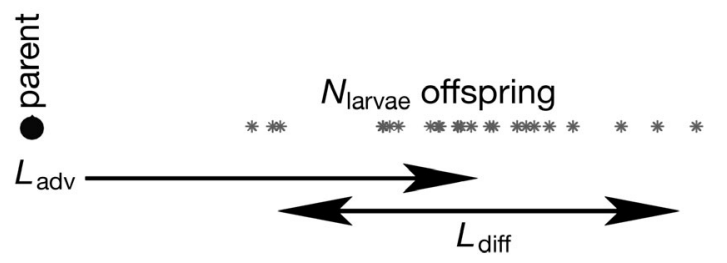

alongshore distance

Fig. 1. Schematic of larval parameters $L_{\mathrm{adv}}, L_{\text {diff, }}$ and $N_{\text {larvae }}$ showing position of a single parent and offspring left by that parent after a lifetime. Asterisks indicate a potential distribution of larval recruitment locations consistent with these values of $L_{\text {adv }}$ and $L_{\text {diff. }}$. See Table 1 for definitions of parameters

stochastic current fluctuations would diffuse the larvae. Byers \& Pringle (2006) quantify these transport parameters as a function of larval behavior, inter- and intra-seasonal current variability and the interaction of current variability and multiple spawning events. They describe how these parameters may be estimated in regions of complex, spatially varying coastal circulation. In order to draw order-of-magnitude conclusions for typical organisms, we present from Byers \& Pringle (2006) a simple estimate of $L_{\mathrm{adv}}$ and $L_{\mathrm{diff}}$ for an organism with simple larval behavior in an ocean whose circulation statistics vary little over the dispersal distance of a larva. Following Siegel et al. (2003), it can be shown that larvae which remain in the plankton for a time $T_{\mathrm{m}}$ will move on average a distance:

$$
L_{\mathrm{adv}}=U T_{\mathrm{m}}
$$

where $U$ is the alongshore flow averaged over the time the larvae are in the plankton. Not all plankton will move the same distance, however, due to random fluctuations in currents associated with eddies, fluctuating winddriven currents and other sources of temporal variability. These fluctuations lead to the spreading of larval settlement locations, and the standard deviation of the larval settlement position (from Siegel et al. 2003) will be:

$$
L_{\text {diff }}=\left(\sigma^{2} \tau_{\mathrm{L}} T_{\mathrm{m}}\right)^{0.5}
$$

where $\sigma$ is the standard deviation of the alongshore currents and $\tau_{\mathrm{L}}$ is the Lagrangian timescale of the fluctuations of the mean alongshore currents, all defined at the depth or depths that the larvae reside. $U$, $\sigma$ and $\tau_{\mathrm{L}}$ are well-characterized properties of the coastal ocean, given an understanding of the vertical distribution of the larvae. Siegel et al. (2003) find that these simple estimates perform well in Central California. A comparison of dispersal distance of many species by Shanks et al. (2003) has found that Eq. (1) overestimates dispersal distance for many species. However, the linear relation between time in plankton and dispersal distance found in Eq. (1) remains valid in this comparison. All of the parameters described above are a function of the location of the parents. This perspective is analo- gous to a Lagrangian reference frame in fluid dynamics (see Siegel et al. 2003) and must be kept firmly in mind when interpreting the results presented below.

Some of the individuals in the population carry allele(s) that give the individual a fitness advantage over other individuals. This is quantified with a selection coefficient $s$ against all other alleles in the population, as in Hartl \& Clark (1997). The models below will be for alleles in haploid organisms or alleles at mitochondrial loci. They will also apply to alleles in the nuclear (diploid) genome as well, if the selective advantage is additive, but the derivations below would have to be altered in the case of overdominance or higher-order gene interactions. In the comparison to observed clines presented below, it must be remembered that the required selection is not necessarily imposed on the genetic markers that actually identify the cline; the individual, not the gene, could be in this case the unit of selection. See Barton \& Hewitt (1989) for a discussion of this and its implications for the retention of other novel diversity.

In order to illustrate and confirm some of the results below, it is useful to have an individual-based numerical model of this idealized organism. The computer model is a straightforward implementation of the organism described above. The habitat in the computer is a one-dimensional array of settlement sites, each of which can hold one adult, and each adult produces $N_{\text {larvae }}$ propagules that move away from the parent as described above. The larval dispersal kernel used is Gaussian. However, the results below are not sensitive to this under typical circumstances, as described in Byers \& Pringle (2006). If the larvae land on a site inhabited by an adult, they die. If several larvae arrive at the same site at the same time, one is randomly chosen as a survivor, with a weighting appropriate for selection, which results in a logistic density dependence. Thus, in the numerical model, selection enters through competition, a form of soft selection (sensu Wallace 1975), though the theory discussed below is developed for both soft and hard selection mechanisms. The code for the model was written in MATLAB and FORTRAN, and is available from the authors.

\section{RESULTS}

\section{Ephemeral nature of a cline between neutral alleles in a uniform advective environment}

To understand how a gradient in allele frequency can be maintained against a mean current in an organism such as described above, we first examine the be- 
havior of such a gradient between 2 neutral alleles in a spatially uniform coastal ocean with a spatially uniform habitat. To do so, a population of the idealized organism described above is separated into 2 groups that differ only by a selectively neutral 'marker' allele, with one allele (or allele class) found in one portion of the habitat, and the other allele predominant in the remaining 'downstream' portion of habitat (Fig. 2). The population density of the entire domain is at its carrying capacity. The progeny of an offspring inherit the allele from their parent.

Individuals with either allele have the same dispersal characteristics, with their larvae moving on average $L_{\text {adv }}$ downstream with a standard deviation of $L_{\text {diff }}$. In Fig. 2, the temporal evolution of this cline is shown. While both allelic populations move downstream, the upstream allele, maintained under the same conditions that maintain the population along the coast (see Byers \& Pringle 2006), rapidly displaces the downstream allele, which is then lost from the population.
The cline moves downstream because, in this region with a saturated habitat, each adult leaves on average only 1 offspring to replace an adult that has just died. This propagule is, on average, $L_{\text {adv }}$ downstream from the adult and so the cline moves, on average, a distance $L_{\text {adv }}$ downstream over a single lifetime. For organisms with relatively long planktonic larval stages $\left(T_{\mathrm{m}}\right.$ : many days or weeks) mean dispersal distances are often tens to hundreds of kilometers per generation (Kinlan \& Gaines 2003, Shanks et al. 2003). For such organisms, a cline between 2 neutral alleles in a spatially uniform domain would sweep through a typical species' geographic range in a few tens to hundreds of generations. For most species, this is a short time compared to either the time it takes to generate a cline through isolation by distance and random genetic drift, or the time since Pleistocene glaciation, a commonly proposed cause of historical allopatry.

However, the downstream alleles in Fig. 2 could persist in the domain if the adults carrying these alleles produced, on average, enough surviving offspring. If this occurred, the stochastic component of larval dispersal could return sufficient offspring to the location of their parents to maintain the downstream allele in the domain. In the next sections, we discuss how the individuals with the downstream allele might be able to produce $>1$ surviving offspring and we define explicit criteria for the persistence of the downstream alleles in the domain.

\section{When can an allele be retained in a region?}

To understand the persistence of a cline in gene frequency along a coastal ocean, it is first necessary to understand when an allele introduced into the center of a uniform coastal habitat with a mean alongshore current will persist. This ocean has alongshore uniform hydrodynamics and habitat and thus $L_{\text {adv }}, L_{\text {diff }}, H_{\text {dens }}$ and $N_{\text {larvae }}$ remain constant along the coast. Individuals carrying the allele are introduced into the habitat at a density much less than the maximum population density that could persist in the habitat, but in large enough numbers that the allele is unlikely to be lost through stochastic fluctuations in gene frequency.

To understand the persistence of the introduced allele, we use a result from Byers \& Pringle (2006) for the persistence of a population. They find that the descendants of an individual of a species intro-
Fig. 2. Evolution of a cline between 2 selectively neutral alleles (grey and black) in the numerical model. Larvae move on average $L_{\text {adv }}=$ $100 \mathrm{~km}$ downstream to the right each generation, with dispersion around that mean of $L_{\text {diff }}=150 \mathrm{~km}$. Habitat is saturated, so each adult leaves, on average, 1 surviving offspring 
duced into an empty habitat, regardless of allelic composition, will persist and be retained in that area, and will grow to populate that area, if:

$$
\ln \left(N_{\text {larvae }}\right)>L_{\text {adv }}^{2} /\left(2 L_{\text {diff }}^{2}\right)
$$

Eq. (3) is valid as long as the domain into which the species is introduced is much larger than $L_{\text {diff }}$ and $L_{\mathrm{adv}}$, and if the dispersal kernel is not leptokurtic, which would make it easier for the introduced species to persist (Byers \& Pringle 2006). When Eq. (3) and these criteria are met, enough larvae are returned to the location of the parents by the stochastic component of larval dispersal ( $\left.L_{\text {diff }}\right)$ to replace adult mortality, despite the downstream transport of most larvae by the mean currents ( $\left.L_{\mathrm{adv}}\right)$.

The dynamics of the retention of a small number of alleles introduced into a population in which they are initially scarce are identical but for one change, for the underlying question is similar: when can the descendents of an allele introduced into a region persist in that region, despite the tendency of the mean currents to transport most copies of the allele downstream? The only change is that the relevant growth parameter is not the number of offspring an adult would leave behind in a region in which the species is scarce ( $\left.N_{\text {larvae}}\right)$, but is instead $N_{\text {allele, the number }}$ of copies of the allele carried by surviving and successfully recruiting offspring, for each copy present a lifetime earlier when the allele is scarce. Thus, the criterion for the retention of the allele introduced into a population is:

$$
\ln \left(N_{\text {allele }}\right)>L_{\text {adv }}^{2} /\left(2 L_{\text {diff }}^{2}\right)
$$

This criterion is explicitly tested in a numerical model below. When this criterion is met, enough copies of an allele successfully recruit to the location of the introduction by the stochastic component of larval transport, such that the population of the allele is maintained there; indeed, the distribution of the allele would tend to extend upstream (Byers \& Pringle 2006) despite the transport of the average larvae downstream. This can be seen in Fig. 3b, in which the introduced allele grows in frequency at and upstream of the location of introduction. If the criterion is not met, the total number of copies of the allele in the population would still tend to increase, but this increase would be insufficient to maintain the allele frequency at the point of introduction. Thus the density of the allele at the point of introduction would decay to zero, the mean location of the introduced alleles would drift downstream, and eventually all copies of the allele would be lost off

the downstream edge of the domain. This is seen in Fig. 3a and in Fig. 2.

The criterion above explains only how an allele introduced into the middle of a coastal ocean could persist. It does not explain how the growth in the number of copies of allele ( $\left.N_{\text {allele }}\right)$ could come about, or how, if Eq. (4) is met, the downstream allele in a cline does not spread upstream and homogenize gene frequency in the upstream direction. In the following section, we focus on the dynamics of an allele in a population that is at the carrying capacity of the environment everywhere. In this case, selection alone can increase $N_{\text {allele }}$ and a spatial gradient in selection is needed to allow a cline to persist but not spread upstream. In the subsequent section, we examine the population dynamics of a region of alongshore variability in habitat or circulation and find a different mechanism for the persistence of a cline.

\section{What selection is needed to allow a cline to persist in a uniform advective environment?}

Gradients in selection have often been invoked to explain the persistence of clines in systems with extensive gene flow (Endler 1977, Hare et al. 2005 and many others). However, we show below that in the presence of non-random components of larval dispersal and uni- 
form habitat, there is a minimum level of selection needed to allow a cline to persist. To do so, we examine the model of a benthic organism given above in the limit that the habitat's carrying capacity is alongshore uniform, and the species has been present for long enough that its population has reached the carry capacity. In this limit, we quantify the minimum level of selection needed to allow an allele to persist and find that it depends sensitively on the mean $\left(L_{\text {adv }}\right)$ and stochastic $\left(L_{\text {diff }}\right)$ components of larval dispersal distance. In order to do so, the increase in the number of copies of an allele that is rare in the population per existing copy of the allele per lifetime, the $N_{\text {allele }}$ given above, is calculated as a function of the selection coefficient $S$ (see Hartl \& Clark 1997) against all other alleles in a population in which the population is habitat limited everywhere. It is then shown with a numerical model that when there is selection for an allele sufficient to satisfy Eq. (4) in, and only in, a downstream portion of a coastal ocean, a cline in allele frequency can persist in the coastal ocean.

In order to calculate $N_{\text {allele }}$ from the selection $s$, we assume that the population in the habitat is limited by the scarcity of habitat, but the model could be easily extended to some other density-dependent process. We also assume that the allele of interest is scarce in the population, for as discussed above and in Byers \& Pringle (2006), this is the limit of interest for retention. $N_{\text {allele }}$ can be written:

$N_{\text {allele }}=$ (larvae per adult per lifetime)

$\times$ (success in competition with other larvae)

$\times$ (fraction of habitat free)

'larvae per adult per lifetime' is defined above as $N_{\text {larvae }}$ and is net of mortality in the larval pool, after settlement and before reproductive competency. $N_{\text {larvae }}$ is defined for an adult that carries the allele with less selective advantage. The 'fraction of habitat free' accounts for the fact that in this simple model, larvae that try to settle where there is an adult will die. The fraction of habitat free is represented by the parameter $\gamma$. If the larvae were to have sufficient behavior to avoid habitat occupied by adults and to seek out nearby available habitat, $\gamma$ would be 1 .

The remaining term of Eq. (5) captures the relative reproductive success of adults with the allele of interest and is where selection enters the equation. It can represent either soft or hard selection mechanisms. The coefficient $s$ is the selection coefficient against other alleles and is defined so that if an adult that does not carry the allele of interest produces $X$ larvae that would successfully recruit, an adult that does carry the allele of interest produces $X /(1-s)$ larvae that successfully recruit. This term can represent an ability to outcompete other larvae for habitat, in which case it re- presents the likelihood that a single larva carrying the allele of interest arriving at an open piece of habitat can succeed in competition against $N_{\text {others }}$ larvae without the allele. Or the term can represent a fractional increase in fecundity or survival in the larval or juvenile pool. Assuming that there are on average $N_{\text {others }}$ other larvae competing for the same piece of open habitat, the success in competition with other larvae is:

$$
\frac{1}{1+(1-s) N_{\text {others }}}
$$

The increase in the number of the selected allele can now be written:

$$
N_{\text {allele }}=\left(\gamma N_{\text {larvae }}\right) /\left\{\left[1+(1-s) N_{\text {others }}\right]\right\}
$$

We assume that the favored allele is scarce in the population, so $N_{\text {others }}$ in the above equation can be eliminated by noting that if there is no selection for the allele $(s=0)$, it will not increase in the population and $N_{\text {allele }}$ is $1 . N_{\text {others }}$ can then be found by setting $s=0$ and $N_{\text {allele }}=1$ in Eq. (7). This solution for $N_{\text {others }}$ can then be substituted into Eq. (7) to give:

$$
N_{\text {allele }}=\left(\gamma N_{\text {larvae }}\right) /\left[(1-s) \gamma N_{\text {larvae }}+s\right]
$$

In the limit of $s<<1$ and $\gamma N_{\text {larvae }}>>1$, this can be written as:

$$
N_{\text {allele }}=1+s
$$

If Eq. (8) is substituted into the criterion for the persistence of an allele in the middle of a population (Eq. 4), it reduces to:

$$
\begin{gathered}
S>L_{\text {adv }}^{2} /\left(2 L_{\text {diff }}^{2}\right) \text { if } s<<1 \text { and } \gamma N_{\text {larvae }}>>1 \\
\ln \left(\gamma N_{\text {larvae }}\right)>L_{\text {adv }}^{2} /\left(2 L_{\text {diff }}^{2}\right) \text { if } \mathrm{s} \rightarrow 1
\end{gathered}
$$

When Eq. (10) is true, an allele introduced into the middle of an alongshore-uniform domain will grow in number and fill the domain, because the increase in the number of copies of the allele due to selection is sufficient to offset the tendency of advection to transport the allele downstream. Note that if $L_{\text {adv }}^{2} /\left(2 L_{\text {diff }}^{2}\right)$ is large enough and $N_{\text {larvae }}$ small enough, an allele can be lost downstream even if it is very strongly selectively favored $(s \approx 1)$.

The derivations of these results in Byers \& Pringle (2006) were only tested in a domain in which the ecological and physical parameters did not vary in the alongshore direction. However, for a cline in allele frequency to persist, Eq. (10) cannot be true everywhere, for if it were, the downstream allele would take over the entire domain. We hypothesize that if the selection for the allele only satisfies Eq. (10) in a downstream portion of the domain, the cline will not move upstream and will occur immediately downstream of the location where selection becomes strong enough to satisfy 


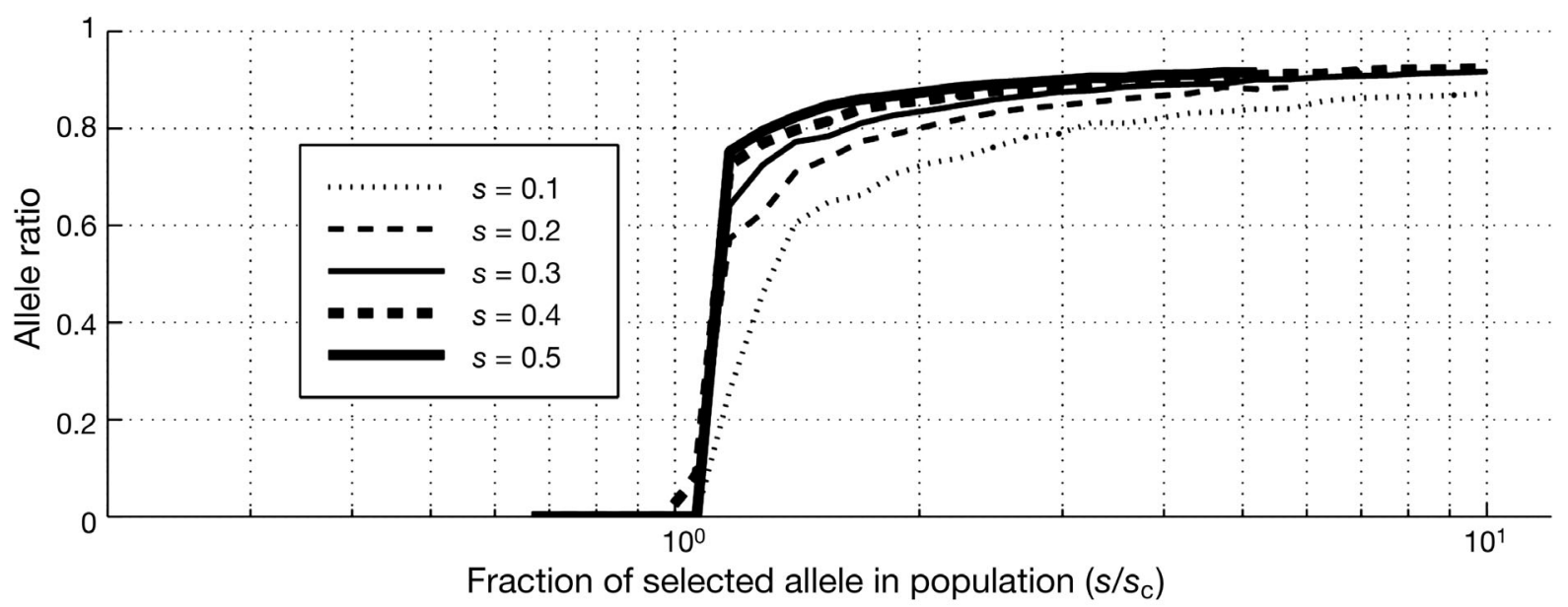

Fig. 4. Allele frequency of favored allele in downstream portion of model domain, as seen in Fig. 5. The allele is only favored in downstream portion of species range. Allele frequency shown as function of selection $s$ normalized by $s_{\mathrm{C}}$, the minimum selection needed to satisfy Eq. (10). Where frequency of allele is zero, selection was insufficient to retain the cline, and downstream allele was flushed out of habitat. Where density is finite, selection was sufficient to maintain the cline against mean current. Model was initialized with favored allele in downstream half of domain and model was run until allele frequency had stabilized. Species modeled had $L_{\text {diff }}$ of $200 \mathrm{~km}$ and $L_{\text {adv }}$ is varied to change critical selection needed to allow retention. In these runs $\gamma \approx 1.0$ for modeled species is semelparous and spawns at end of lifetime

Eq. (10). When we test this conjecture in the numerical model of the population, it is found to be correct (Figs. 4 \& 5), as long as the downstream region is large

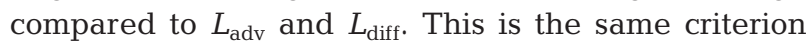
found for the validity of Eq. (3) in Byers \& Pringle (2006) and references therein.

Thus for a cline to persist in a uniform environment in which the population is limited by habitat scarcity, there must be a gradient in selection so that Eq. (10) is satisfied only in the downstream portion of the domain. In this limit, the selection needed for a cline to persist depends on the larval dispersal parameters $L_{\mathrm{adv}}$ and

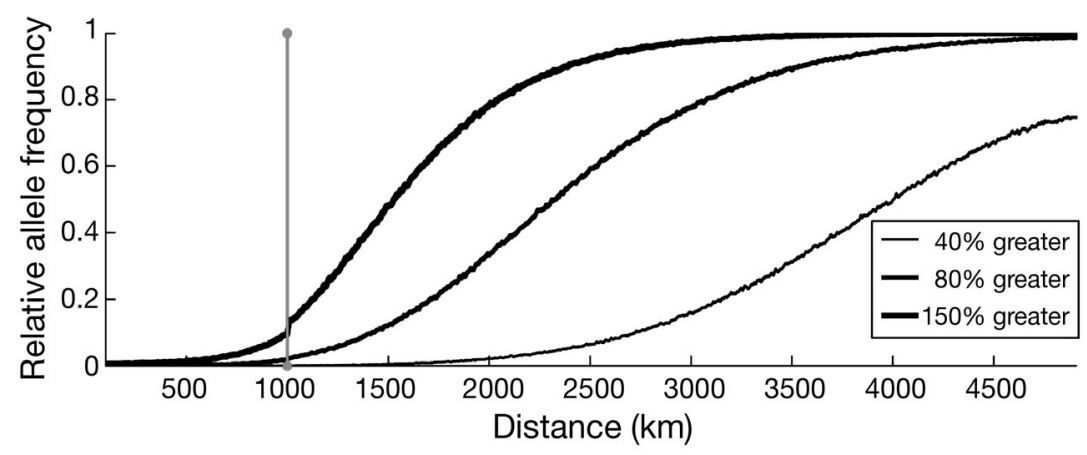

Fig. 5. Frequency of an allele which is selectively favored downstream (right) of the $1000 \mathrm{~km}$ point of domain, but not upstream of that point, in the model. Selection is 40,80 and $150 \%$ greater than that needed to satisfy Eq. (10). Initial and boundary conditions are as in Fig. $5 . L_{\text {adv }}=100 \mathrm{~km}, L_{\text {diff }}$ $=200 \mathrm{~km}$ and critical value of selection to allow cline to persist is $S=0.1958$ for semelparaous species
$L_{\text {diff. }}$ This will be discussed further below. However, it is first necessary to examine another limit, in which a cline is maintained by an alongshore variation in either ocean circulation or habitat, and the subsequent reduction in habitat scarcity.

\section{Persistence of a cline allowed by alongshore variation in habitat and transport}

In the preceding section, the number of successful offspring an individual could leave was limited by the scarcity of habitat, and thus $N_{\text {allele }}$ was driven by selection. The number of copies of an allele left by an adult can also be increased if each adult is able to leave multiple offspring because the environment is not, at least locally, habitat limited. These regions of enhanced reproductive success can be areas in which $N_{\text {allele }}$ satisfies Eq. (4) and thus can support a persistent cline. In order to understand these dynamics we examine the model in the limit where alongshore variation in carrying capacity or larval transport produces regions of enhanced reproductive success.

Of course, if a species' total population is in steady state, the average adult will only leave a single offspring behind after its death. However, this need not be true 

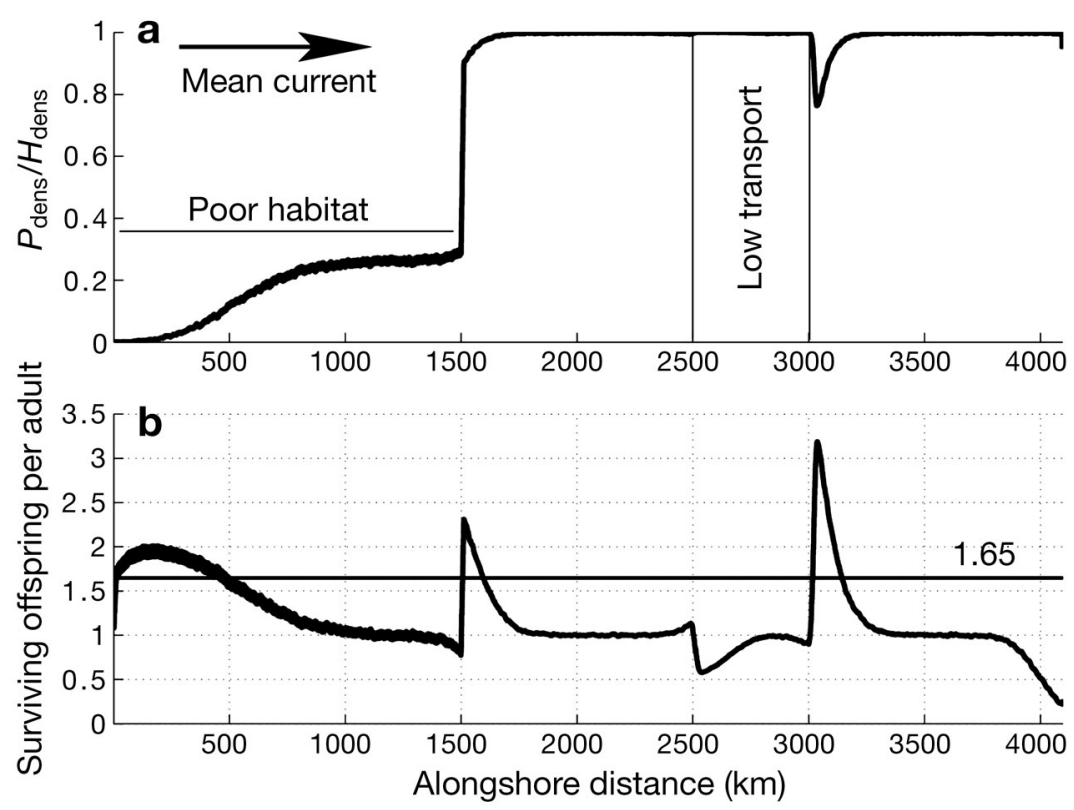

Fig. 6. (a) Distribution of population density as calculated by numerical model for a finite habitat with a region of poor habitat capable of carrying only $1 / 3$ of normal population between 0 and $1300 \mathrm{~km}$, and a region of weak ( $10 \%$ of normal) alongshore flow and dispersion between 2500 and $3000 \mathrm{~km}$. Mean current flowing to right; $L_{\mathrm{adv}}=L_{\text {diff }}=400 \mathrm{~km}$ outside region of reduced alongshore flow and dispersion. (b) Actual reproductive success in surviving offspring per adult. Horizontal line at 1.65: critical rate to satisfy Eq. (4)

everywhere in the species range, for some regions can be source populations in which each adult has above-average reproductive success, while other regions can be sink regions (Fig. 6). This pattern of sources and sinks can be seen most clearly in considering the entire range of a species in an environment with unidirectional mean alongshore current. Adults in the center of the domain leave, on average, 1 successful child per lifetime, for where the population growth is limited by density dependence children can only survive to replace adults that have died. Adults near the downstream edge of the species range largely fail to reproduce successfully as their progeny are, on average, swept downstream and beyond suitable habitat. On the other hand, adults on the upstream edge of the species range have a greater reproductive success rate because the larvae produced at the upstream edge of the species range serve to repopulate both this region and the area immediately downstream of it.

Similar regions of enhanced reproductive success can also form any place in the domain where the supply of larvae from upstream is insufficient to fully supply the downstream region with larvae, and so the population is below the local carrying capacity of the habitat. This can occur either when larval transport from upstream is interrupted, or when available habitat increases downstream. For example, the carrying capacity of the habitat triples downstream of the $1300 \mathrm{~km}$ point of Fig. 6. Reproductive success is high here and the population is recruit-limited, for the larvae produced in this region feel little competition from the small number of larvae advected in from the poor habitat upstream. Another region of high reproductive success and reduced population occurs downstream of a region of reduced larval transport between 2500 and $3000 \mathrm{~km}$ because few larvae are exported from there to settle downstream.

In these regions of enhanced reproductive success, Eqs. (4) \& (3) can be satisfied. Thus, upon their death, adults in these regions will usually be replaced with recruits that originated in the same region. These areas are thus retention areas (sensu Byers \& Pringle 2006) and alleles in these regions, including any novel alleles that may arise in these regions, will tend to persist. Thus we can treat these retention regions as populations, in the classical population genetic sense. This will allow us to apply classical population genetic results to these areas and to analyze the retention of a cline at these locations in terms of the migration between populations upstream and downstream of the change in habitat or circulation.

At the upstream edge of the species domain there is no immigration from further upstream and the usual balance between mutation and drift will govern allelic diversity. In those regions of high reproductive success in the interior of the species range, allelic diversity will be governed not only by these processes, but also by the immigration of small numbers of individuals from upstream. It is necessary to quantify the processes that create regions of high reproductive success and limit immigration from upstream in order to understand their relative importance. The parameter $f_{\text {migrate }}$ is the ratio of the larval supply from upstream to the supply that would occur if habitat and circulation in the retention zone extended infinitely upstream, so that a smaller number indicates fewer larvae from upstream settle in the region; at the upstream edge of the species range, $f_{\text {migrate }}=0$. Where the reduced larval supply is formed by an interruption in the alongshore transport of larvae, $f_{\text {migrate }}$ is the fraction of larvae which can pass through the interruption. Where the reduced larval supply is caused by poor upstream habitat, $f_{\text {migrate }}$ is the ratio between the carrying capacity upstream of and within the retention region. When the population in the region of enhanced reproductive success is close to 
the carrying capacity, then $f_{\text {migrate }}$ also approximates the ratio of migrants to local recruits that settle in the region of enhanced reproductive success (see Appen$\operatorname{dix} 1)$.

If $f_{\text {migrate }}$ is much less than the mutation rate of a gene, classical population genetic results suggest that the migration rate would be insufficient to homogenize the populations upstream and downstream of the region of high reproductive success. A cline could exist there solely due to stochastic fluctuations in the frequency of neutral alleles (Nagylaki 1978, Hartl \& Clark 1997). However, if $f_{\text {migrate }}$ is greater than the mutation rate, some selection for a downstream allele would be necessary to maintain it against immigration from upstream.

The relative importance of immigration and selection in a deme has been extensively studied in cases of asymmetric dispersal (e.g. Kawecki \& Holt 2002). In these studies, the frequency of the favored allele in the population receiving migrants is $1-s / m$ for $s \ll 1$, where $m$ is the ratio of migrants to local recruits in the retention region. Since $m \approx f_{\text {migrate, }}$, the frequency of the

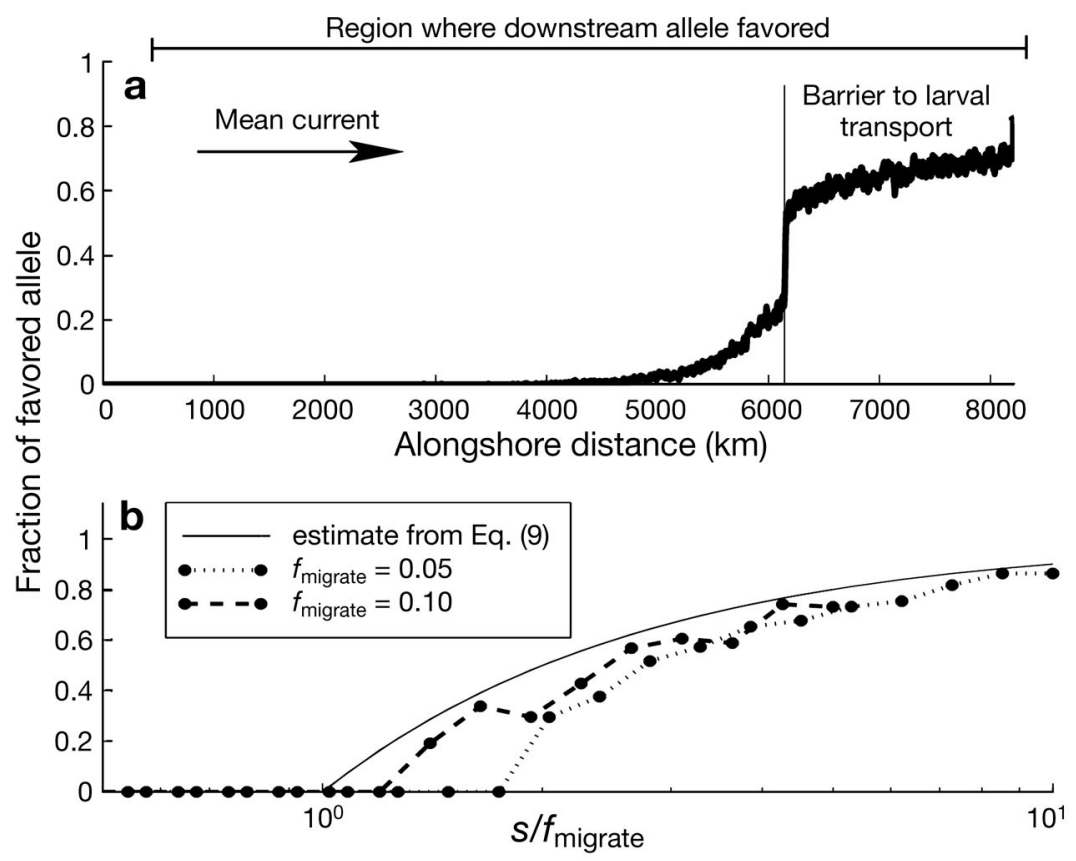

Fig. 7. (a) Steady-state frequency of favored allele in model. Allele is favored downstream of $500 \mathrm{~km}$; barrier to larval transport is at $6100 \mathrm{~km}$. Organisms with favored allele are released just downstream of barrier at start of model run and model is run until populations reach steady state. Favored allele selected by $s=0.16 ; 95 \%$ of larvae prevented from crossing barrier to larval transport in either direction. (b) Frequency of favored allele in retention zone downstream of barrier to larval transport as a function of selection for downstream allele for both $f_{\text {migrate }}=0.05$ and 0.1 and prediction of this ratio from Eq. (11). Selection is scaled by $f_{\text {migrate }}$ so that all curves lie upon each other. $L_{\mathrm{adv}}=200 \mathrm{~km}, L_{\text {diff }}=300 \mathrm{~km}, N_{\text {larvae }}=6$. See Appendix 1 for discussion of the validity of Eq. (11) and associated errors favored allele in the retention region downstream of the variation in habitat or circulation is approximately:

$$
\text { Allele frequency }=\left(1-f_{\text {migrate }} / s\right)
$$

for $f_{\text {migrate }} \ll 1, s \ll 1$, where $s$ is the selection coefficient against all other alleles in the retention zone. This is illustrated in Fig. 7 and derived more formally, along with a discussion of its limitations, in Appendix 1. When $f_{\text {migrate }}>S$, the favored allele will be lost from the system and the cline will disappear. When $f_{\text {migrate }}<s$ the allele and the cline will persist where there is the change in alongshore larval transport or habitat.

At this point, it may seem that there is little difference between the classical problem of 2 populations exchanging migrants and an advective coastal environment with alongshore variation in larval transport or habitat. In both, reduction in larval transport between 2 regions increases the ability of a cline to persist by reducing the migration between the 2 populations, e.g. Slatkin (1985), Hare \& Avise (1996), Lenormand (2002). However, directional dispersal of the species changes the system in 2 fundamental ways.

Firstly, in a system with directional dispersal, if the reproductive success in the downstream region is reduced to the point that Eq. (4) is not satisfied, no non-zero $f_{\text {migrate }}$ will allow the favored allele to persist only in the downstream region. Population dynamics, in particular the reproductive success of adults in the downstream region, fundamentally govern the ability of an allele to be retained in an advective environment, and the enhanced reproductive success is enabled by alongshore variation in circulation or habitat. Secondly, directional dispersal prevents an allele from flourishing everywhere it is selectively favored, as can be seen in Fig. 7. In this figure, an allele is favored in the downstream $9 / 10$ of the domain and there is a reduction of larval transport in the midst of the region where the downstream allele is favored. Since selection for the allele is less than that required to satisfy Eq. (10), the cline is not able to spread upstream of the location of the interruption of larval transport. The cline is instead pinned to the region of alongshore variability in larval transport, even though the downstream allele is favored upstream of that point. Thus clines will persist not where an allele begins to be favored, but where there is a retention zone driven by alongshore changes in circulation or habitat, or where the selection exceeds the minimum set by Eq. (10). 


\section{DISCUSSION}

\section{Maintenance of phylogeographic boundaries in a species with long planktonic larval duration}

To maintain population genetic boundaries in the coastal ocean with alongshore uniform circulation and habitat, there must be a gradient in selection for the downstream allele. There must be sufficient selection downstream of the cline to retain the downstream allele against advection, and insufficient selection upstream of the cline to prevent the cline from moving upstream. In such a relatively simple coastal ocean, and for species with uncomplicated spawning and larval behavior, we can estimate the critical level of selection at the cline. Substituting Eqs. (2) \& (1) into Eq. (10) produces an expression for this critical selection threshold as a function of the circulation parameters and larval duration:

$$
\begin{array}{cc}
s>\frac{U^{2} T_{m}}{2 \sigma_{u}^{2} \tau_{L}} & \text { if }\left(U^{2} T_{\mathrm{m}}\right) /\left(2 \sigma^{2} \tau_{\mathrm{L}}\right)<<1 \\
s \rightarrow 1 & \text { if }\left(U^{2} T_{\mathrm{m}}\right) /\left(2 \sigma^{2} \tau_{\mathrm{L}}\right)>>1
\end{array}
$$

Increased mean currents $(U)$ and reduced variability in the currents $(\sigma)$ will tend to increase the selection needed to retain an allele, while a decreased time in plankton $\left(T_{\mathrm{m}}\right)$ will reduce the selection needed to retain an allele.

For most coastal oceans, the strength of $U$ is comparable to the strength of the fluctuations of the current $(\sigma)$, so the term $U^{2} /\left(2 \sigma^{2}\right)$ in Eq. (12) is of O(1) (Winant et al. 1987 for central California; Beardsley et al. 1985 for the Mid-Atlantic Bight; Hickey \& Banas 2003 for the northeast Pacific; Boicourt et al. 1998 for the Gulf of Mexico). Thus the magnitude of the selection needed to retain an allele will depend on the ratio $T_{\mathrm{m}} / \tau_{\mathrm{L}}$. Where $T_{\mathrm{m}}$ is much greater than the timescale of current fluctuations $\tau_{\mathrm{L}}$ it will take strong selection to allow a cline to persist in place. If $T_{\mathrm{m}}$ is much shorter than $\tau_{\mathrm{L}}$ it will take moderate levels of selection to allow an allele to spread upstream and only moderate selection gradients to preserve a cline in place. $\tau_{\mathrm{L}}$ in most coastal oceans is on the order of a few days (Davis 1985, Seigel et al. 2003) and so moderate values of $s$ will suffice in species whose larvae disperse in the plankton for less than a few days. However, if larvae with simple behavior are in the plankton for much more than several days, the selection coefficient needed to allow a cline to persist increases dramatically. Kingsolver et al. (2001) found that the median of observed levels of selection on quantitative traits in natural populations is about $s=0.16$, though with a long tail of much higher selection levels. For single-locus patterns of selection, a number of examples in marine populations are available for which $s$ is apparently much higher (reviewed in Eanes 1999). For most species with a $T_{\mathrm{m}}$ longer than a few days, clines can only be retained along a coast with relatively uniform current and habitat by high levels of selection.

A cline can also persist in a region where the mean current goes locally to zero or becomes small, allowing $L_{\text {adv }}$ to become much less than $L_{\text {diff }}$ and thus allowing weak levels of selection to retain an allele (Eq. 10). $L_{\text {adv }}$ may also become small due to larval or spawning strategies that enhance retention. Byers \& Pringle (2006) describe several such strategies, such as the spawning of larvae in multiple seasons with oppositely directed mean currents to reduce $L_{\text {adv }}$. Hare et al. (2005) illustrate the importance of other mechanisms that may reduce transport across a region, all of which can lead to significant genetically controlled clines.

Additionally, even for species with long planktonic durations, a cline can persist where there is a reduction in larval pressure from upstream due to alongshore variation in circulation or habitat. These regions of persistence will not be fully captured in analyses that neglect alongshore variation in population dynamics. In these regions, the selection needed to retain the downstream allele in a cline is a function only of the strength of the disruption of larvae supply from upstream (Eq. 11) and is not a function of larval duration. This allows clines to persist with modest gradients of selection even for species with long $T_{\mathrm{m}}$. If $f_{\text {migrate }}$ is less than the mutation rate for an allele, the cline can persist with no selection. Given the low median levels of selection seen by Kingsolver et al. (2001), this model suggests that most clines observed in species with long $T_{\mathrm{m}}$ are associated with reduced larval supply from upstream.

Of course, the magnitude of the limitation of upstream larval supply $f_{\text {migrate }}$ might be a function of larval duration and dispersal distance. For example, $f_{\text {migrate }}$ could be small for larvae in the surface waters where there is a region of mean offshore transport of the surface waters, such as in an upwelling zone. In this case, a longer larval duration would increase the likelihood of larvae being lost offshore. This would decrease $f_{\text {migrate }}$ and allow the cline to persist with less selection. The interruption of alongshore larval supply can also be driven by a region of poor habitat, which supplies few larvae to a downstream region. For larval dispersal distances less than the size of the region of poor habitat, no larvae could pass through this region from upstream and $f_{\text {migrate }}$ would not be a function of the dispersal distance and larval duration. However, once the larval duration increased and dispersal distances $L_{\mathrm{adv}}$ or $L_{\mathrm{diff}}$ exceeded the size of the region of poor habitat, $f_{\text {migrate }}$ would increase abruptly and the selection needed to preserve a cline would increase. 
Thus our model suggests that, for species with long planktonic larval durations and large ratios of $L_{\mathrm{adv}}$ to $L_{\text {diff }}$, clines will occur either in regions of elevated reproductive success driven by reduced larval immigration from upstream, or the cline will be maintained by levels of selection much larger than the median values found by Kingsolver et al. (2001), or both.

\section{Central California cline in Balanus glandula; a cline and long planktonic larval duration}

Balanus glandula is a well-studied barnacle present along much of the Pacific coast of North America (Newman et al. 1980). Sotka et al. (2004) describe a cline in both nuclear and mitochondrial allele families for B. glandula across a narrow region of the Central Californian coast. This phylogeographic transition spans ca. $500 \mathrm{~km}$ of coastline between Pacific Grove, California, and Cape Mendocino, California (Sotka et al. 2004). For this analysis, we chose parameters appropriate for this species and for the oceanographic conditions in this region. Using current meter data, we calculate in Appendix 2 that $L_{\text {adv }}$ is ca. $290 \mathrm{~km}$ and $L_{\text {diff }}$ $240 \mathrm{~km}$.

In the absence of any alongshore variation in habitat or circulation the selection needed to retain an allele here would be about $s=0.7$, if each adult barnacle could produce many surviving offspring in a sparsely populated area. This selection is much larger than the median seen by Kingsolver et al. (2001), though is within the range of selection coefficients observed. If the selection for the downstream allele was greater than this in the cline, and less to the north, a selection gradient alone could explain the cline.

However, it is likely that the circulation in this region would tend to greatly reduce the selection needed to allow the cline to persist, and thus reducing the required selection to a more typically observed value. Physical theory suggests that the change in the continental shelf width between Pt. Reyes and Monterey Bay will cause a reduction of alongshore transport between these 2 points, with the excess transport on the shelf moving offshore in the surface Ekman layer (Pringle 2002). The reduction in the alongshore transport is expected to scale as the ratio of the shelf width at the 2 locations and thus there should be a 5- or 10fold reduction in alongshore transport. Evidence from floats drogued to follow the surface waters (Sotka et al. 2004) supports this theory. Floats released to the south of the cline in late winter tend to move along the coast and beach on the coast near Monterey Bay or to the south (e.g. winter float tracks in Winant et al. 2003), while floats released to the north of the cline tend to move offshore between Pt. Reyes and Pt. Arena, and then stay offshore, never to return to the coast where a larva could settle (Davis 1985, Sotka et al. 2004). Nearshore observations in Monterey Bay support the idea that alongshore transport in that area is opposite to the prevailing mean, and drives offshore transport (Storlazzi et al. 2003). Thus the physical environment in the area of the cline provides the interruption of the larval transport needed to allow a cline to persist. Studies of barnacle population dynamics in the region of the cline support the idea that the population is limited by larval supply, and not by habitat (Roughgarden et al. 1988 and references therein), suggesting that the interruption in larval supply has set the stage for reproductive success, and thus the retention of alleles in this region.

Similar interactions between local adaptive processes and variation in nearshore physical oceanography have been hypothesized to drive phenotypic and other macroevolutionary patterns in other marine systems (Taylor \& Hellberg 2003, Cowen et al. 2006).

\section{Present location and historical origin of a cline}

Evidence suggests that the age of a cline at a particular location can be much less than the age of the genetic differences that form the cline (Felsenstein 1975, Endler 1977). The differentiation of the allelic classes which form clines in the Balanus glandula population appears to have formed $>10^{5} \mathrm{yr}$ BP (Wares $\&$ Cunningham 2005). However, the change in shelf width that Pringle (2002) posits as the cause of the interruption of larval transport at the present cline location between Monterey and San Francisco Bay only exists at present sea levels. When the sea level was $\sim 100 \mathrm{~m}$ lower in the last ice age (Graham et al. 2003), the shelf would have vanished, and the proximate physical cause of the cline would disappear. Thus the present mechanism for fixing the cline in place may have only existed for the last $10000 \mathrm{yr}$.

However, this model suggests that a cline could persist even without refugia. If the present interruption of larval transport in central California were to be removed, the cline would be swept downstream until it re-established at the next location along the coast in which larval transport were interrupted, or one of the other physical or biological mechanisms for cline formation discussed above existed. If the mean currents experienced by larvae were always in the same direction, and the retention zones are sufficiently separated as to prevent larval dispersal from the downstream to upstream retention zone, then the cline would ratchet downstream from one extent retention zone to the next as the retention zones came and went. For these reasons, tracking phylogeographic boundaries in marine populations necessarily involves understanding con- 
temporary forces that limit the movement of individuals or their larvae. Many of these transitions, along with biogeographic transitions, may have originated due to historical events but their current location may not be the site at which the transition originated.

\section{Relation of the above theory to existing phylogeographic theory}

It is well established that the location and strength or shape of a cline can be modified by stochastic allele frequency drift (Felsenstein 1975, Slatkin \& Maruyama 1975), and there is a large body of literature that looks at the effects of selection and genetic drift in demes that undergo asymmetric migration (e.g. Nagylaki 1978, Lundy \& Possingham 1998, Lebreton et al. 2000, Kawecki \& Holt 2002, Lenormand 2002). Some of the results derived here are foreshadowed in these papers and the links can now be made explicit. Nagylaki (1975) showed that an allele that was favored in a region could be lost if there was a large amount of migration into that region from areas in which the allele was not selected for. Nagylaki (1978) pointed out that it was easier to maintain an allele in a deme which generated a net outflow of migrants than in one which was a sink for migrants, and Kawecki \& Holt (2002) argued that selection in sink regions would be relatively ineffective (cf. Lebreton et al. 2000). Lundy \& Possingham (1998) argued that a deme would only contribute to preventing the fixation of an allele if it were a source of migrants.

However, to place the results of these papers in the context of a coastal ocean, it is necessary to identify the mechanisms that form populations or demes there. The tendency has been to label regions of relatively uniform allele frequency as a population. This is not appropriate, for in most of these regions any novel allelic diversity or stochastic fluctuation in allele frequency that arises will be swept downstream by the mean currents and will not contribute to the evolution of allelic diversity at that point. We can only discover the boundaries between populations by considering the spatial structure of habitat and circulation.

Regions downstream of a reduction in larval supply can retain stochastic fluctuations in allele frequency and novel alleles that arise and the region of enhanced reproductive success can be considered a population. These regions also export larvae downstream so they are source regions for the downstream population. As Kawecki \& Holt (2002) argue, selected alleles are relatively easily retained in these regions, even if there is some migration into the population from upstream. If immigration of larvae into this region is less than the mutation rate in the population, a cline can exist even in the absence of selection. At the upstream edge of the species range, where there can be no immigration of larvae from upstream, allele frequency will change as one would expect for a population with symmetric dispersal (e.g. Kimura 1968).

The sink populations are those downstream of the regions of enhanced reproductive success, in which density dependence limits the realized growth rate of the population. At each of these regions, the population is maintained by immigrants from upstream, and the region maintains the population downstream of it by exporting its larvae downstream. As predicted by Nagylaki (1978), Kawecki \& Holt (2002) and others, it takes a very large selection coefficient to maintain an allele in the population in these sink regions (Eq. 10). Without strong selection, allele frequency at downstream regions is set by the gene frequency $L_{\mathrm{adv}}$ upstream, until in the end it is set by the allele frequency at an upstream region of enhanced reproductive success.

In these downstream regions, the substitution rate will not equal the mutation rate. Mutants will arise at a rate proportional to the population size, but will on average be distributed further downstream each generation; given enough mutants over large distances with large population sizes, a stochastic equilibrium defined by mutation and upstream diversity will hold. This would predict increased levels of allelic diversity in downstream 'sink' habitats (Wares et al. 2001, AlRabab'ah \& Williams 2004). However, as seen in Fig. 2 and in the associated text, the distribution of any mutant allele would shift downstream by $L_{\text {adv }}$ each lifetime. For species with mean planktonic dispersal distances of tens to hundreds of kilometers, and species ranges of thousands of kilometers in size, any mutant allele which arises outside of the downstream edge will be lost off the downstream edge of the domain in a few tens to hundreds of generations, a time perhaps insufficient to allow significant allelic diversity to develop (e.g. Hoskin 2000).

\section{Marine protected areas, fisheries and the transport of clines}

The genetic homogeneity of a population along the coast has been used to argue that the population should be treated as a single population or 'stock' for the purposes of fisheries management. The genetic homogeneity has been assumed to indicate that the population is panmictic and thus individuals in any part of the population can replenish other parts of the population. However, as described above, this is not true in downstream sink regions, whose population is maintained by immigration from upstream. 
In such a population, harvesting could even encourage the formation of sub-populations if the harvesting in the interior of the domain created a region of small population in the midst of a range, thus creating the interruption in larval transport needed to create locally high realized reproductive success and thus the local retention of allelic diversity. Conversely, sufficient harvesting could destroy a cline and cause the upstream genome to invade downstream. Clines can be maintained by weak selection in local regions where the realized growth rate is high enough to satisfy Eq. (4), as discussed above. If a fisheries reduces the growth rate by harvesting reproducing adults, the growth rate could fail to satisfy (Eq. 4), preventing retention and allowing the cline to be swept downstream. The local population would then be taken over by the upstream population, which, while less adapted to local conditions, is anchored by some more robust retention zone farther upstream.

\section{Directional dispersal in other environments}

Similar genetic dynamics to those described above will exist in any system in which the dispersal of propagules is anisotropic, with a preferred direction of dispersal, for example in populations with wind-driven dispersal (Kawecki \& Holt 2002, Al-Rabab'ah \& Williams 2004) or populations in the open ocean. Qualitatively, the above results hold. However, the quantitative results for retention and the maintenance of clines described above will only hold if the dimension of the population range perpendicular to the direction of preferred dispersal is small enough such that the population can disperse across it in $\mathrm{O}(1)$ generations. If it is not, the quantitative results described above must be modified to include tendency of isotropic dispersal normal to the direction of preferred dispersal to reduce the growth rate at a fixed point in space, a calculation similar to the classic calculations of the relative importance of dispersal and growth made in Kierstead \& Slobodkin (1953).

\section{SUMMARY}

The population genetics of the coastal ocean has long been understood as resulting from the interplay of selection, random genetic drift, and migration (e.g. Palumbi 2004 and references therein). The interplay of these mechanisms in the coastal ocean for organisms with planktonic larval stages is simultaneously governed by ocean circulation and local population dynamics. Thus it is only by taking currents and population dynamics into account that the population dynamic analysis of demes and sub-populations can be mapped onto a continuous coastal ocean, with little in the way of natural boundaries. In doing so, it has become clear that the maintenance of polymorphism in these systems is modified by advection and local population dynamics. A cline will not usually be located where an allele becomes favored. Instead, the cline will persist either where the selection for an allele begins to satisfy (Eq. 10) or where an alongshore variation in habitat or circulation produces a fractional reduction in the upstream larval supply greater than the selection for an allele or the mutation rate. For species with long planktonic larval durations our model suggests that the latter is more likely to set the location of a cline.

Acknowledgements. This is GLOBEC submission 324 and was funded by NSF grant OCE-0219709 to J. Pringle. Thanks go to R. K. Grosberg, S. T. Small and R. J. Toonen for comments on the manuscript.

\section{LITERATURE CITED}

Al-Rabah'ah MA, Williams CG (2004) An ancient bottleneck in the Lost Pines of central Texas. Mol Ecol 13:1075-1084

Avise JC (1994) Molecular markers, natural history and evolution. Chapman \& Hall, New York

Avise JC (2000) Phylogeography: the history and formation of species. Harvard University Press, Cambridge, MA

Barnes H, Barnes M (1956) The general biology of Balanus glandula Darwin. Pac Sci 10:415-421

Barton NH, Hewitt GM (1989) Adaptation, speciation and hybrid zones. Nature 341:497-503

Beardsley RC, Chapman DC, Brink KH, Ramp SR, Schlitz R (1985) The Nantucket Shoals Flux Experiment (Nsfe79). 1. A basic description of the current and temperature variability. J Phys Oceanogr 15:713-748

Boicourt WC, Wiseman WJ, Valle-Levinson A, Atkinson LP (1998) Continental shelf of the southeastern United States and Gulf of Mexico: in the shadow of the western boundary current. In: Allan R, Brink KH (eds) The sea, Vol 11. John Wiley \& Sons, New York, p 135-183

Brown SK, Roughgarden J (1985) Growth, morphology, and laboratory culture of larvae of Balanus-Glandula (Cirripedia, Thoracica). J Crustac Biol 5:574-590

Byers JE, Pringle JM (2006) Going against the flow: retention, range limits and invasions in advective environments. Mar Ecol Prog Ser 313:27-41

Cowen RK, Paris CB, Srinivasan A (2006) Scaling of connectivity in marine populations. Science 311:522-527

Davis RE (1985) Drifter observations of coastal surface currents during CODE - the statistical and dynamical views. J Geophys Res C 90:4756-4772

Eanes WF (1999) Analysis of selection on enzyme polymorphisms. Annu Rev Ecol Syst 30:301-326

Endler JA (1977) Geographic variation, speciation, and clines. Princeton University Press, Princeton, NJ

Felsenstein J (1975) Genetic drift in clines which are maintained by migration and natural selection. Genetics 81 : 191-207

Graham MH, Dayton PK, Erlandson JM (2003) Ice ages and ecological transitions on temperate coasts. Trends Ecol Evol 18:33-40 
Grosberg RK (1982) Intertidal zonation of barnacles: the influence of planktonic zonation of larvae on vertical distribution of adults. Ecology 63:894-899

Hare MP, Avise JC (1996) Molecular genetic analysis of a stepped multilocus cline in the American Oyster (Crassostrea virginica). Evolution 50:2305-2315

Hare MP, Guenther C, Fagan WF (2005) Nonrandom larval dispersal can steepen marine clines. Evolution 59: 2509- 2517

Hartl DL, Clark AG (1997) Principles of population genetics. Sinauer, Sunderland, MA

Haydon DT, Crother BI, Pianka ER (1994) New directions in biogeography. Trends Ecol Evol 9:403-406

Hickey BM, Banas NS (2003) Oceanography of the US Pacific Northwest coast and estuaries with application to coastal ecology. Estuaries 26:1010-1031

Hines AH (1978) Reproduction in three species of intertidal barnacles from central California. Biol Bull 154:262-281

Hoskin MG (2000) Effects of the East Australian Current on the genetic structure of a direct developing muricid snail (Bedeva hanleyi, Angas): variability within and among local populations. Biol J Linn Soc 69:245-262

Irwin DE (2002) Phylogeographic breaks without geographic barriers to gene flow. Evolution 56:2383-2394

Kawecki TJ, Holt RD (2002) Evolutionary consequences of asymmetric dispersal rates. Am Nat 160:333-347

Kierstead H, Slobodkin LB (1953) The size of water masses containing plankton blooms. J Mar Res 12:141-147

Kimura M (1968) Genetic variability maintained in a finite population due to mutational production of neutral and nearly neutral isoalleles. Genet Res 11:247-269

Kingsolver JG, Hoekstra HE, Hoekstra JM, Berrigan D and 5 others (2001) The strength of phenotypic selection in natural populations. Am Nat 157:245-261

Kinlan BP, Gaines SD (2003) Propagule dispersal in marine and terrestrial environments: a community perspective. Ecology 84:2007-2020

Lebreton JD, Khaladi M, Grosbois V (2000) An explicit approach to evolutionarily stable dispersal strategies: no cost of dispersal. Math Biosci 165:163-176

Lenormand T (2002) Gene flow and the limits to natural selection. Trends Ecol Evol 17:183-189

Lentz SJ, Chapman DC (1989) Seasonal differences in the current and temperature variability over the northern California shelf during the coastal ocean dynamics experiment. J Geophys Res C 94:12571-12592

Lundy IJ, Possingham HP (1998) Fixation probability of an allele in a subdivided population with asymmetric migration. Genet Res 71:237-245

Nagylaki T (1975) Conditions for the existence of clines. Genetics 80:595-615

Nagylaki T (1978) Clines with asymmetric migration. Genetics 88:813-827
Newman WA, Abbott DP (1980) Cirripedia. In: Morris RH, Abbott DP, Haderlie EC (eds) Intertidal invertebrates of California. Stanford University Press, Stanford, CA, p 504-535

Palumbi SR (1994) Genetic-divergence, reproductive isolation, and marine speciation. Annu Rev Ecol Syst 25: $547-572$

Pringle JM (2002) Enhancement of wind-driven upwelling and downwelling by alongshore bathymetric variability. J Phys Oceanogr 32:3101-3112

Roughgarden J, Gaines S, Possingham H (1988) Recruitment dynamics in complex life cycles. Science 241:1460-1466

Shanks AL, Grantham BA, Carr MH (2003) Propagule dispersal distance and the size and spacing of marine reserves. Ecol Appl 13:S159-S169

Siegel DA, Kinlan BP, Gaylord B, Gaines SD (2003) Lagrangian descriptions of marine larval dispersion. Mar Ecol Prog Ser 260:83-96

Slatkin M (1985) Gene flow in natural populations. Annu Rev Ecol Syst 16:393-430

Slatkin M, Maruyama T (1975) Genetic drift in a cline. Genetics 81:209-222

Sotka EE, Wares JP, Hay ME (2003) Geographic and genetic variation in feeding preference for chemically defended seaweeds. Evolution 57:2262-2276

Sotka EE, Wares JP, Barth JA, Grosberg RK, Palumbi SR (2004) Strong genetic clines and geographical variation in gene flow in the rocky intertidal barnacle Balanus glandula. Mol Ecol 13:2143-2156

Storlazzi CD, McManus MA, Figurski JD (2003) Long-term, high-frequency current and temperature measurements along central California: insights into upwelling/relaxation and internal waves on the inner shelf. Cont Shelf Res 23:901-918

Taylor MS, Hellberg ME (2003) Genetic evidence for local retention of pelagic larvae in a Caribbean reef fish. Science 299:107-109

Wallace B (1975) Hard and soft selection revisited. Evolution 29:465-473

Wares JP, Cunningham CW (2005) Diversification predates glaciation in Balanus glandula. Biol Bull 208:60-68

Wares JP, Gaines SD, Cunningham CW (2001) A comparative study of asymmetric migration events across a marine biogeographic boundary. Evolution 55:295-306

Winant CD, Beardsley RC, Davis RE (1987) Moored wind, temperature, and current observations made during Coastal Ocean Dynamics Experiments-1 and Experiment2 over the northern California continental-shelf and upper slope. J Geophys Res C 92:1569-1604

Winant CD, Dever EP, Hendershott MC (2003) Characteristic patterns of shelf circulation at the boundary between central and southern California. J Geophys Res Oceans 108, doi:10.1029/2001JC001302 
Appendix 1. Maintenance of a cline against migration from upstream

To quantify the relative importance of immigration and selection in the retention region downstream of alongshore variation in the circulation or habitat, we examine the allele frequency of an allele which is favored downstream of the change in circulation or habitat. The relative frequency of this allele in the retention region downstream of the interruption is $R_{\mathrm{d}}$, and the total number of alleles in that region $P_{\text {reten }}$. We wish to find $R_{\mathrm{d}}$ as a function of the selection $\mathrm{s}$ against all alleles but the favored downstream allele and the fractional reduction in the ratio of larvae entering the retention region from upstream $f_{\text {migrate }}$. As part of the derivation, it is useful to define $N_{\mathrm{m}}$, the number of copies of the other alleles that enter the retention region to compete for habitat. Parameters defined only in this appendix are not listed in Table 1.

The number of copies of alleles which return to the retention region each lifetime will scale as $N_{\text {larvae }} P_{\text {reten }}$. We assume that the immigration $N_{\mathrm{m}}$ is much smaller than $N_{\text {larvae }} P_{\text {reten, }}$ and so the number of copies of the favored downstream allele that compete for available habitat each lifetime scales as $N_{\text {larvae }} P_{\text {reten }} R_{\mathrm{d}}$, and the number of copies of the other alleles are $N_{\mathrm{m}}+N_{\text {larvae }} P_{\text {reten }}\left(1-R_{\mathrm{d}}\right)$. From Eq. (6) we can then write the fraction of the larvae carrying downstream allele which compete successfully for the available habitat as:

$$
\left(N_{\text {larvae }} P_{\text {reten }} R_{\mathrm{d}}\right) /\left\{N_{\text {larvae }} P_{\text {reten }} R_{\mathrm{d}}+(1-\mathrm{s})\right.
$$$$
\left.\left[N_{\mathrm{m}}+N_{\text {larvae }} P_{\text {reten }}\left(1-R_{\mathrm{d}}\right)\right]\right\}
$$

If the allele frequency is in steady state, the fraction of the larvae carrying the downstream allele that recruit successfully must equal the fraction of the allele in the population and so we can solve for $R_{\mathrm{d}}$ by setting $R_{\mathrm{d}}$ equal to Eq. (A1) and solving for $R_{\mathrm{d}}$. This results in:

$$
\begin{gathered}
R_{\mathrm{d}}=1-\left[N_{\mathrm{m}} /\left(N_{\text {larvae }} P_{\text {reten }}\right)\right](1-s) / s \\
\approx 1-\left[N_{\mathrm{m}} /\left(P_{\text {reten }} N_{\text {larvae }}\right)\right] / s \quad \text { if } s<1
\end{gathered}
$$

This is the classic result that the balance of immigration and selection is governed by the ratio of selection $s$ to the fractional migration rate $m=N_{\mathrm{m}} /\left(N_{\text {larvae }} P_{\text {reten }}\right)$ when $s<1$.

In order to get an estimate of how $N_{\mathrm{m}} /\left(N_{\text {larvae }} P_{\text {reten }}\right)$ varies with the strength of $f_{\text {migrater }}$ we assume that $P_{\text {reten }}$ is of the same order as the carrying capacity of the retention zone. This assumption is valid when $N_{\text {larvae }}$ is large, as in Fig. 6. Then, since without the interruption in larval transport, $N_{\text {larvae }} P_{\text {reten }}$ larvae from upstream would attempt to settle in the retention zone per lifetime, with the interruption there would be $N_{\mathrm{m}}=f_{\text {migrate }} N_{\text {larvae }} P_{\text {reten }}$ larvae from upstream. The fraction of the downstream allele in the retention zone is then:

$$
R_{\mathrm{d}}=1-f_{\text {migrate }} / s \text { if } s \ll 1
$$

Eq. (A3) becomes less than zero when the selection rate is not greater than $f_{\text {migrate, }}$ which implies that the cline would not be maintained. Where it is greater, a cline can be maintained. The abundance of the downstream allele increases as one moves farther downstream from the retention zone, for selection will serve to increase the abundance of the allele as it is swept downstream from the retention zone.

The derivation for Eq. (A3) above makes 3 approximations that limit its accuracy. First, it assumes that there are none of the favored allele upstream of the variation in habitat or circulation, so that all migrants from upstream contain the disfavored alleles. Second, it assumes that $P_{\text {reten }}$ is equal to the carrying capacity of the habitat in the retention zone, while it must actually be somewhat less to allow the enhanced reproductive success needed to preserve the cline. Third, it assumes that larvae spawned in the retention zone are as likely to return to the retention zone as those from immediately upstream. The first assumption would increase $R_{\mathrm{d}}$, while the second and third decrease it. The exact impact of these assumptions depends on the exact nature of the alongshore variation of circulation and habitat; however, in cases we have modeled it is relatively minor, changing the selection needed to allow retention by $<30 \%$. Eq. (A3) is compared to model runs in which $f_{\text {migrate }}$ is set to 0.05 and 0.10 by removing $\left(1-f_{\text {migrate }}\right)$ of the larvae crossing a point $3 / 4$ of the model domain from the upstream edge. The downstream allele is favored in the downstream $9 / 10$ of the model domain. The models are then run for a large number of differing values of $S$, and the concentration of the downstream allele is shown in Fig. 7. Eq. (A3) is seen to capture the variability of $R_{\mathrm{d}}$ with $s$ well. 
Appendix 2. Balanus glandula dispersal and life history

\begin{abstract}
Balanus glandula, like many other decapod crustaceans, has a planktonic larval form that feeds and develops in the water column for 3 to $4 \mathrm{wk}$ before becoming competent to settle (Brown \& Roughgarden 1985). B. glandula broods larvae from February to October with the majority (ca. 90\%) being released early and ca. $10 \%$ of the release from August to September; settlement is then during September/October (Hines 1978). The brooding cycles for B. glandula are similar for populations in British Columbia, central California, and southern California (Barnes \& Barnes 1956), and late larval stage individuals are found within the top $0.5 \mathrm{~m}$ of seawater (Grosberg 1982).

$L_{\text {adv }}$ and $L_{\text {diff }}$ for Balanus glandula in the vicinity of the cline can be estimated from the mid-shelf alongshore currents measured as part of the Coastal Ocean Dynamics Experiment in the northern part of the cline, between Pt. Reyes and Pt. Arena. Lentz \& Chapman (1989) estimate a mean alongshore current $U$ in the spring and early summer mid-shelf surface waters of $14 \mathrm{~cm} \mathrm{~s}^{-1}$ southward with a standard deviation $\sigma$ of $24 \mathrm{~cm} \mathrm{~s}^{-1}$, and a fall mean current of $7 \mathrm{~cm}$ $\mathrm{s}^{-1}$ northward with a standard deviation of $18 \mathrm{~cm} \mathrm{~s}^{-1}$. Davis (1985) estimates that the Lagrangian timescale $\tau_{\mathrm{L}}$ was about $3 \mathrm{~d}$. The net larval dispersal combining the early- and late-
\end{abstract}

season larval releases was calculated as in Byers \& Pringle (2006), in which the values of $L_{\text {adv }}$ and $L_{\text {diff }}$ for each spawning event is weighted appropriately by the fraction of larvae released in each event, resulting in an estimate of $L_{\mathrm{adv}}=287$ $\mathrm{km}$ and an $L_{\text {diff }}=240 \mathrm{~km}$. No attempt was made to compensate for different mortality rates in the late spring versus late summer, for there is insufficient information to do so, though it is likely that more larvae are lost offshore in the spring upwelling season as surface waters are transported offshore by Ekman transport (Roughgarden et al. 1988). A relatively higher mortality in the spring than in the fall due to offshore advection would increase $L_{\text {diff }}$ and decrease $L_{\text {adv }}$.

Shanks et al. (2003) find for many species that behavior reduces the mean dispersal distance, though it remains linearly related to the time in plankton. How this would affect the results for Balanus glandula is unclear for 2 reasons. First, there are not sufficient studies of the behavior of $B$. glandula larvae to determine the magnitude of any behavior-related reduction in larval transport. Second, the effects on retention and allele persistence depend on the ratio of $L_{\text {adv }}$ to $L_{\text {diff, }}$ and it is even less clear how this ratio might be affected by behavior. See Byers \& Pringle (2006) for more details on the interaction of behavior with retention.
Editorial responsibility: Steven Morgan (Contributing Editor), Bodega Bay, California, USA
Submitted: May 2, 2006; Accepted: September 5, 2006

Proofs received from author(s): March 28, 2007 